\title{
DE LA UNIFICACIÓN DE TEORÍAS \\ A LA INTEGRACIÓN DE DISCIPLINAS ${ }^{*}$
}

EDNA SUÁREZ

FACULTAD DE CIENCIAS

UNIVERSIDAD NACIONAL AUTÓNOMA DE MÉxICO

\section{Introducción}

Tradicionalmente se ha asumido que la ciencia es una empresa unificada en un sentido epistemológico. En qué sentido debe entenderse dicha unificación, dada la aparente diversidad de la ciencia, es algo que ha recibido diferentes respuestas. El ejemplo clásico de un proyecto de unificación es el del modelo de reducción teórica de Nagel (1961), estrechamente ligado a la concepción neopositivista de la ciencia. Según este proyecto las relaciones entre las diferentes teorías científicas tienden a constituirse $-\mathrm{y}$ por tanto pueden modelarse- como relaciones de reducción. Detrás de ese modelo se encontraba el supuesto de que teorías más fundamentales - las teorías de la física, en última instancia, podrían explicar las teorías y leyes menos fundamentales de la ciencia mediante la construcción de estructuras teóricas cada vez más abarcantes. Pese a las numerosas críticas que ha recibido el modelo de Nagel y, de hecho, pese a su abandono por la enorme mayoría de los filósofos de la ciencia, la concepción de la unificación de la ciencia por reducción ha persistido como una idea influyente (Ruse, 1973; Schaffner 1967, 1993; Waters 1990) o incluso como un supuesto de quienes ven, en la imposibilidad de la reducción, un argumento contra la posible unificación de la ciencia (por ejemplo Hull, 1974; Rosenberg, 1994). ${ }^{1}$

Mi intención en este trabajo no es, sin embargo, discutir el proyecto de unificación de la ciencia por reducción, sino plantear una alternativa, no necesariamente incompatible, a la idea de que la unificación o, mejor aún, la integración de la ciencia es, fundamentalmente, una relación que esencialmente involucra teorías. La motivación de un enfoque de este tipo

* Agradezco a Sergio F. Martínez M. los comentarios a una versión previa de este trabajo. Varias de las ideas que introduzco aquí serán desarrolladas después en un trabajo común.

1 El libro de Dupré (1993), The Disorder of Things, constituye un esfuerzo por problematizar y minar los supuestos metafísicos que se encuentran detrás de esta idea tan poderosa $\mathrm{e}$ influyente de la ciencia moderna. 
proviene de controversias y perspectivas de la ciencia muy diferentes a las que motivan el proyecto de unificación por teorias. Comenzaré presentando el enfoque que pretendo defender refiriéndome brevemente al tipo de controversias y temas que están detrás de un enfoque "integracionista". 2 Veamos.

En buena parte como resultado del impacto de la sociología y la historia de la ciencia en el planteamiento de problemas filosóficos, en los últimos años se ha puesto una atención creciente al estudio de las "culturas locales". A partir de estos estudios ha surgido la idea de que la ciencia tiene una naturaleza fragmentada. Por naturaleza fragmentada de la ciencia me refiero a la idea de que en la ciencia coexisten diferentes tipos de objetivos epistémicos, los cuales no se limitan a la construcción de teorías y, por tanto, requieren del establecimiento de prácticas y habilidades no estrictamente "teóricas". Una primera versión de esta idea se encuentra en autores como Hacking (1983), Galison (1987) y Pickering (1989), quienes han señalado la importancia de lo que aquf llamo tradiciones experimentales. Hacking (1992), por ejemplo, ha hablado de la ciencia como de una estructura "parchada" (patchy) y Lenoir (1993) ha sostenido la idea de que la ciencia tiene una estructura "disgregada". Esta idea, que es central para la propuesta que presento en este artículo, se irá desarrollando en apartados posteriores.

Ahora bien, la idea de la fragmentación de la ciencia no está libre de problemas. En particular, esta idea parece llevarnos de la mano a la ineludible conclusión de que la ciencia no es más que un grupo de culturas locales débilmente conectadas entre sí (quizás con un cierto parecido de familia) y que, en última instancia, el conocimiento científico no es más que el resultado de restricciones e interacciones locales. .Así pues, la tendencia - por demás bienvenida - de los estudios de la ciencia a realizar análisis de caso desde una perspectiva sociológica e histórica, parece conducirnos a tirar al niño junto con el agua de la bañera. Por un lado esta tendencia actúa con completa indiferencia frente a cuestiones que han tenido gran importancia en la historia de la filosofía, por ejemplo, el carácter privilegiado del conocimiento científico, la naturaleza de la evidencia científica o la posibilidad de establecer relaciones de explicación entre diversas áreas del conocimiento. Y, por otro, esta tendencia prejuzga la propia historia y sociología de la ciencia al suponer en sus estudios de caso aquello (lo local o fragmentado) que es lo que intenta mostrar.

Es en este contexto donde vuelve a adquirir relevancia la cuestión de en qué sentido la ciencia constituye $\longrightarrow$ no- un sistema unificado de conoci-

2 Voy a hablar de integración y no de unificación para marcar terminológicamente la distancia entre el tipo de enfoque que pretendo desarrollar aquí y el tipo de enfoque que unifica, asociado sobre todo con la filosofia neopositivista. 
miento. La solución no puede consistir en ser indiferente ante los resultados y consecuencias de los estudios histórico-sociológicos, máxime cuando éstos constituyen una de las zonas más vitales de reflexión sobre la ciencia. Sin embargo, bajo la perspectiva de tales estudios se corre el riesgo de un relativismo o balcanización extremos (por ejemplo, Collins, 1988), que se apoyan en el carácter exclusivamente local de los análisis. El reto, pues, es el siguiente. ¿Cómo entender a la ciencia como una empresa fragmentada, de manera tal que podamos reconocer y asimilar los importantes resultados de los estudios de caso, y a la vez ser capaces de darle sentido a la ciencia como empresa unificada o unificable de modo que.nos permita responder a cuestiones filosóficas fundamentales? Esto es, ¿cómo responder a la pregunta de qué es lo que unifica a la ciencia, cuando un buen número de estudios destacan las ventajas del enfoque localista en el estudio de la misma?.

Para elaborar una respuesta que me permita reconocer tanto el aspecto fragmentado de la ciencia como su aspecto integrable, parto de la idea de que la fragmentación de la ciencia puede entenderse como resultado de la existencia de diferentes tipos de tradiciones científicas. Al hablar de tipos de tradiciones científicas (en el sentido de Martínez, 1993a, 1993b, 1995 que desarrollaré adelante) me refiero a la construcción histórica de diferentes tipos de prácticas y habilidades alrededor de distintos tipos de objetos y fines epistémicos, que no necesariamente son de tipo teórico. La idea de tipos de tradiciones se apoya en el reconocimiento (sustentado empíricamente por numerosos estudios de caso) de que el conocimiento puede corporalizarse en objetos y fines epistémicos tan diversos como son las técnicas y artefactos tecnológicos de un laboratorio o las clasificaciones naturales. Así, a diferencia de los proyectos de unificación de la ciencia que se centran en el establecimiento de relaciones entre teorías, el proyecto de integración que quiero proponer se refiere a diferentes interacciones sociales, técnicas (materiales) y cognitivas entre diferentes tradiciones. Más aún, en ciertas circunstancias histórico-sociales tales interacciones permiten la conformación y consolidación de nuevas disciplinas científicas, de manera que el tipo de integración del conocimiento científico al cual me voy a referir debe ser visto como un proceso de integración de tradiciones en disciplinas y no como un problema de unificación de teorías.

La argumentación de este trabajo se dividirá en varias secciones. En la primera presento y critico algunas propuestas en las que la integración de la ciencia es vista fundamentalmente como el establecimiento de una relación entre teorías. En las secciones siguientes presento mi propuesta para enfrentar el problema de la integración de la ciencia a través de la constitución de disciplinas científicas; esta presentación se apoya en un caso que he estudia- 
do a fondo, el de la disciplina biológica llamada evolución molecular. ${ }^{3} \mathrm{Mi}$ propuesta busca apoyar la idea de que el conocimiento se articula en objetos diversos que se construyen en diferentes tipos de tradiciones (sección 2), las cuales -bajo ciertas circunstancias- establecen entre sí una variedad de interacciones no exclusivamente teóricas (sección 3). La consolidación de una disciplina involucra un tipo de integración cognitiva, y no "meramente social", que se expresa en el concepto de dominio de una disciplina (sección 4); dicho concepto me permitirá abundar en la naturaleza de una integración del conocimiento científico que parte del reconocimiento de la fragmentación de la ciencia y, en ese sentido, es compatible con los objetivos que busco reconciliar. Por último, en la sección 5 intento hacer explícitas algunas de las consecuencias y ventajas de esta noción de integración de la ciencia.

\section{La concepción ortodoxa de la unificación científica}

Como seqñalé, el problema de en qué sentido la ciencia se encuentra unificada o es unificable, se ha respondido proponiendo diversas maneras en que se da $\longrightarrow$ puede modelarse - la relación entre teorías científicas. Un modelo de la unificación de la ciencia que, como mencioné, tuvo gran influencia hasta hace poco, fue el modelo de reducción teórica de Nagel (1961). En este modelo la unificación de la ciencia se ve como un proceso de reducción lógica en el que intervienen dos tipos de teorías: la teoría reducida y la teoría reductora. Las condiciones formales bajo las cuales Nagel sostenía que podía llevarse a cabo la reducción (la derivabilidad de la teoría reducida a partir de la reductora y la conectabilidad o identidad entre los términos de ambas teorías), la noción que este modelo presupone de lo que es una teoría científica, así como el rechazo paulatino de la concepción neopositivista de la ciencia, condujeron al abandono general de este modelo. Pero si bien el modelo de Nagel ha recibido numerosas críticas y modificaciones a partir de finales de la década de los sesenta (véase Sarkar, 1992, y Suárez, 1996), es indudable que autores con posiciones muy distintas continúan sosteniendo algunos de sus supuestos característicos y, en particular, de la noción de unificación de la ciencia subyacente.

Schaffner $(1967,1993)$, por ejemplo, critica la concepción de las teorías que está implícita en el modelo de Nagel, pero estaría de acuerdo con que el modelo es más o menos adecuado para referirse al proceso de unificación de la ciencia. Por otra parte, autores que defienden la llamada concepción semántica de las teorías, como Rosenberg (1994) o Waters (1990),

3 Este caso es una elaboración de un tema que he desarrollado en mi tesis de doctorado (Suárez, 1996). 
y que difieren en sus conclusiones respecto a la importancia del reduccionismo en biología, parecen aceptar junto con Nagel la relevancia de la reducción teórica para el proyecto de la unificación; Rosenberg piensa que la imposibilidad de la reducción conduce a la disunidad de la ciencia, mientras que Waters opina que el desarrollo de la ciencia parece conducirnos a la construcción de explicaciones (reduccionistas) que unifican diferentes áreas del conocimiento.

Sin embargo, más allá de la discusión en torno a la relación entre reduccionismo y unidad de la ciencia, el supuesto más atrincherado en todos los modelos de unificación es la idea de que ésta es o se basa en una relación - por especificarse- entre teorías. Este supuesto implica, a su vez, una noción restrictiva de la estructura y el papel de las disciplinas científicas en la unificación o integración de la ciencia. Veamos.

Tradicionalmente la filosofía de la ciencia le ha dado mayor importancia al papel de las teorías como articuladoras del conocimiento y como estructuras explicativas. Tanto en las concepciones sintácticas como en las llamadas concepciones semánticas de las teorías, es un supuesto que la estructura de éstas constituye un núcleo más o menos rígido alrededor del cual se articula el conocimiento propiamente científico. ${ }^{4} \mathrm{Al}$ señalar este supuesto no pretendo (como haré ver en la siguiente sección) restar importancia al papel que las teorías y modelos teóricos juegan en la articulación de una gran parte del conocimiento; de hecho, en esto consiste una de sus funciones centrales. Sin embargo, diversos estudios empíricos nos muestran que las teorías no son las únicas estructuras que pueden desempeñar esa y otras funciones que tradicionalmente se les asignan. ${ }^{5}$ Lo que me interesa destacar es que tanto si se cree que las teorías son conjuntos de axiomas a partir de los cuales se derivan leyes de aplicación universal, como si se acepta la idea

4 La importancia de llevar a cabo reconstrucciones racionales de las teorías, uno de los principales objetivos en toda esta tradición, descansa en este supuesto. Pero si se acepta (con sus implicaciones más radicales) que el conocimiento es una construcción histórica en la cual, de manera contingente y relativa a contextos, se van desarrollando explicaciones, conceptos y relaciones entre conceptos, entonces deja de ser un objetivo importante la reconstrucción abstracta de un corte sincrónico de ese proceso.

5 Dice Wimsatt (1976b): "Disputes over the relative importance or primacy of structure and function were frequent in 19th century biology, though there now seems to be fairly widespread agreement among biologists that neither is primary, and each affects the other. For philosophy of science in the positivistic tradition, the corresponding question has not even been seriously entertained; there has been an unequivocal emphasis on structure" (p. 213). La distinción que utilizo en el texto entre estructura y función es bastante laxa (en especial si recordamos la complejidad de esta discusión en biología), sin embargo me parece que la idea de Wimsatt sugiere algo importante. Si se reconoce la diversidad de fines epistémicos en la ciencia, podría empezar a reconocerse que diversos tipos de estructuras, algunas más adecuadas que otras, pueden cumplir con la función de alcanzar tales fines. 
de que las teorías son conjuntos de modelos restringidos en sus aplicaciones, el tipo de unificación de la ciencia que debemos esperar, si asumimos las implicaciones y resultados de los estudios históricos y sociológicos, no puede restrigirse a modelar tipos de relaciones interteóricas.

Una de las consecuencias del supuesto de que la unidad de la ciencia consiste $\longrightarrow$ tiende a - una relación entre teorías, es la utilización de una noción extremadamente restrictiva de lo que son, y qué papel juegan, las disciplinas científicas. Bajo el supuesto de la unidad como unidad-teórica a las disciplinas se las considera únicamente como "marcos" en los cuales se desarrollan y justifican las teorías y, por tanto, como estructuras que solamente cumplen un papel de "mediación" en la unificación de la ciencia. En el caso del modelo reductivo, por ejemplo, se sostiene que las teorías de unas disciplinas se derivan de las teorías de otras disciplinas. Además, tanto en la literatura reduccionista (Waters, 1990) como en la de sus críticos (Kitcher, 1984), es común que se identifiquen teorías y disciplinas y que a partir de eso se hable, por ejemplo, de "la reducción de la genética a la biología molecular". 6

A esta concepción -que las más de las veces es implícita-, la llamaré la concepción ortodoxa de las disciplinas. En ella, la cuestión de la estructura de una disciplina se restringe a resolver qué teoría(s) delimita(n) o alrededor de qué teoría(s) se articulan los elementos de la disciplina. El dominio, las leyes y las explicaciones que utilizan y construyen cotidianamente los miembros de una disciplina se ven como cuestiones que se siguen de una teoría o de un conjunto de modelos teóricos.

Llama la atención que buena parte de las diferentes propuestas sobre la estructura de las disciplinas se hayan realizado en el contexto de diversos modelos de integración de la ciencia, entre los cuales destaca el modelo de unificación sin reducción de Darden y Maull (1977). En la propuesta de Darden y Maull la unificación de la ciencia ocurre mediante el establecimiento de relaciones teóricas de tipo no-reductivo entre dos o más campos de investigación. La noción de campo de investigación adquiere importancia para las ideas que me interesa desarrollar, ya que un campo es una estructura

6 La concepción ortodoxa de las disciplinas ha marcado de modo especial el debate en torno a la reducción de la genética clásica por la genética molecular. Diversos autores han señalado (por ejemplo, Hull, 1972, 1974 y Kitcher, 1984) que no existe una teoría ni de la genética ni de la biología molecular y que, por tanto, la relación entre ambas disciplinas-teorías no puede verse como una reducción (deductiva) al estilo de Nagel. Más aún, se ha señalado que existen muchos elementos en la genética clásica (sus técnicas, conceptos o la naturaleza de sus explicaciones) que no pueden reducirse a una teoría de carácter deductivo. Sin embargo, incluso autores como Kitcher (1984), que hablan de la genética como un conjunto de prácticas, incurren en el quid pro quo de hablar indistintamente de teorías y disciplinas (en este caso Kitcher habla de "ciencias"). 
similar a una disciplina aunque más pequeña. ${ }^{7}$ Un campo puede contener una o varias teorías (intrafield theories), pero éstas no son un elemento necesario. Un campo, más bien, se caracteriza por un problema central, un dominio (que son las cosas que se toman como hechos relacionados con el problema central), ${ }^{8}$ factores explicativos generales, objetivos que aportan las expectativas acerca de la solución de los problemas, técnicas, métodos, un vocabulario especial y, con frecuencia, conceptos, leyes y teorías relacionados con el problema central. Un campo, además, tiene un origen, un desarrollo y en ocasiones un momento de extincion; sus elementos pudieron haber existido de manera independiente o formando parte de otros campos, pero para que emerja un nuevo campo éstos deben interaccionar de "manera fructífera" (p. 44).

La propuesta de Darden y Maull reconoce que las relaciones entre teorías y campos no son unívocas ni fácilmente discernibles (esto es, no hay una teoría para cada campo ni cada campo contiene necesariamente una o varias teorías). Pero el estudio de casos históricos de la biología muestra, según estas autoras, que uno de los medios por los cuales ocurre la integración de campos de investigación en la biología consiste en la construcción de teorías locales. A esas teorías que establecen puentes entre campos de investigación muy localizados las llaman teorías intercampos (interfields theories) y se caracterizan porque "comparten un interés en explicar diferentes aspectos de un mismo fenómeno". Así pues, una teoría intercampos, según Darden y Maull, puede verse como la respuesta a un problema o conjunto de problemas que aparecen en uno o varios campos y que no tiene(n) respuesta con los conceptos y técnicas de ese campo. ${ }^{9}$

7 Darden y Maull (1977) reconocen que su noción de campo es muy cercana a la noción de "disciplina" de Stephen Toulmin (op. cit., p. 45), pero es significativo que para ellas (ibid. p. 46) uno de los elementos del concepto de disciplina de Toulmin que no tiene cabida en su concepto es "la experiencia acumulada de los científicos". Este elemento, en cambio, ocupa un lugar importante en mi concepción de las disciplinas a través del papel que le otorgo a las prócticas cientificas que constituyen los tipos de tradiciones científicas.

8 Las nociones de campo y de dominio fueron adoptadas y desarrolladas por estas autoras a partir del trabajo de Shapere (1974 [1984]). Como haré ver en la sección 4, la idea de dominio, que originalmente se refería al dominio de una teoría, puede extenderse de manera fructifera a la idea de dominio de una disciplina.

9 Dicen Darden y Maull (1977): "The existence of such interfield theories has been obscured by analysis such as Nagel's that erroneously conflate theories and fields and see interrelations as derivational reductions" (p. 43-44). Los ejemplos de teorías intercampos que han estudiado Darden y Maull (1977) son el de la teoria cromosómica de la herencia (que integró, en una relación de partes-todo los campos de la citología y la genética), la teoría bioquímica del gen (que integró funcionalmente campos de la genética y la bioquímica) y la teoría de la regulación alostérica (que integró desde una perspeetiva estructural y funcional campos de la bioquímica y la química). 
La caracterización de un campo de investigación desarrollada por Darden y Maull ha sido retomada por diferentes autores, quienes han preferido hablar de disciplinas científicas y de su papel en la integración de la ciencia (Bechtel, 1993; Burian, 1993). Para estos autores el enfoque de Darden y Maull ha sido un buen punto de partida en el análisis de casos de integración en la biología y en la reformulación del concepto de disciplinas. ${ }^{10} \mathrm{Sin}$ embargo, el modelo de Darden y Maull se limita a un tipo de integración que, aunque importante en la historia de la ciencia, no agota las posibilidades de integración de diferentes tipos de conocimiento científico. Ello se debe a que el proyecto de Darden y Maull no reconoce, más que de palabra, la existencia de objetos y fines epistémicos distintos y autónomos de las teorías científicas. Esto es, a pesar de que su definición de campo parece incluir muchos tipos de elementos (técnicas, preguntas, objetivos, métodos, etc.), las autoras no sugieren de qué manera esos diversos elementos coadyuvan al establecimiento de relaciones entre diversas áreas del conocimiento.

En conclusión, el problema con la concepción ortodoxa, que comparten muchas de las propuestas que buscan ser alternativas, es que presupone que epistémicamente una disciplina no es algo más que sus teorías y, por tanto, el problema de la unidad o integración de la ciencia puede modelarse como un problema de relación (o construcción de relaciones) interteóricas. El supuesto central, pues, consiste en creer que una vez que explicamos una relación entre teorías explicamos todo lo que hay que explicar respecto a las relaciones entre disciplinas y, así, respecto al papel que éstas juegan en la unificación del conocimiento científico. Como haré ver más adelante, las disciplinas cumplen un papel importante, pero muy diferente, en la integración del conocimiento científico.

\section{Tipos de tradiciones científicas}

El énfasis en los elementos exclusivamente conceptuales, y particularmente teóricos, de las relaciones entre campos o disciplinas, deja de lado el carácter social e histórico de la integración de la ciencia. Este aspecto ha sido, por el contrario, el foco de atención de numerosos estudios recientes de la ciencia: las actividades cotidianas de los científicos, la constitución y evaluación de los objetos que constituyen el medio material en el cual éstos desarrollan su trabajo y la construcción de evidencias (facts), fenómenos y explicaciones. Como señalé en la introducción, una de las conclusiones más difundidas

10 En este trabajo no analizaré las propuestas de Bechtel (1993) y Burian (1993), autores que en mayor o menor medida han criticado el supuesto de que la integración de la ciencia es una cuestión de relaciones interteóricas. La propuesta de Bechtel es bastante compatible con la idea de disciplinas que desarrollo a continuación. Las ideas de Burian parecen difícilmente asimilables a una concepción filosófica de la ciencia (véase Suárez, 1996 para más detalles). 
de este tipo de estudios es la de que no existe un criterio epistémico único que permita decidir lo que es el conocimiento científico; la ciencia, más bien, es vista como un conjunto de prácticas, de habilidades y de objetos reconocidos y validados como tales por una comunidad (véase Latour, 1987; Collins, 1988, por ejemplo).

La idea de Martínez (1993a, 1993b, 1995), de que en la ciencia existen diferentes tipos de tradiciones científicas, resulta particularmente interesante en este contexto, en buena parte porque permite estructurar los resultados de estos estudios en una perspectiva filosófica. Con la idea de tipos de tradiciones científicas Martínez se refiere a la articulación de diferentes tipos de prácticas y habilidades epistémicas que se han desarrollado históricamente alrededor de diferentes tipos de objetos y fines epistémicos. En particular, este autor ha hablado de tradiciones científicas de tipo teórico, experimental $y$ descriptivista, pero esta lista no es ni busca ser exhaustiva.

Ahora bien, es importante no confundir los tipos de tradiciones científicas con las tradiciones individuales que ejemplifican esos tipos. En la actualidad muchos autores hablan del papel de las tradiciones en la ciencia, pero en general se refieren a "tradiciones individuales", las cuales se entienden como "culturas locales" (por ejemplo Burian, 1993a y Gaudillière, 1993; véase Vicedo, 1995). La caracterización de tradiciones individuales cumple por lo general funciones historiográficas pero no va más allá; esto es, no se reconoce la existencia de diferentes tipos de prácticas como el punto de partida de la caracterización de esas tradiciones, algo que es central en la caracterización de Martínez. Los tipos de prácticas, en este sentido, son la corporalización de distintas capacidades cognitivas que generan y promueven conocimiento. Ese conocimiento se articula a su vez en prácticas novedosas y, al menos en parte, queda implícito en las tradiciones y no se manifiesta en teorías. En efecto, las prácticas evolucionan alrededor de objetos o fines epistémicos tan distintos como son la construcción de teorías o modelos teórico-matemáticos, la estabilización de fenómenos en un laboratorio y la evolución de técnicas experimentales, o la construcción de clasificaciones y árboles filogenéticos. ${ }^{11}$

11 Estos objetos constituyen, como veremos en la sección siguiente, los fines epistémicos primordiales en las tradiciones teóricas, experimentales y descriptivistas de la evolución molecular. Debo señalar que por "teorías" o "modelos teórico-matemáticos" me refiero al tipo de teorías o modelos que caracterizan, por ejemplo, la genética de poblaciones; este tipo de modelos se construye partiendo de suposiciones matemáticas sobre las probabilidades en los cambios de frecuencias génicas. Este tipo de teorías debe distinguirse de lo que llamo modelos de fenómenos, estructuras explicativas con aplicación restringida, que son características de las tradiciones experimentales y en las cuales se busca representar las relaciones causales que existen entre distintos fenómenos y procesos. 
Ahora bien, el concepto de tradición de Martínez busca abandonar el muy extendido supuesto de que la conexión racional entre prácticas y creencias o juicios debe estar mediado por teorías. En la medida en que algunos tipos de prácticas corporalizan conocimiento sin necesidad de mediación teórica entonces - necesariamente- los conjuntos de prácticas que constituyen las tradiciones de una disciplina se encuentran integrados, pero esa integración no puede reducirse a una relación entre teorías. ${ }^{12}$ Así pues, la idea de que existen diferentes tipos de tradiciones científicas nos permite ir más allá de los modelos de integración teórica de la ciencia.

\section{Las disciplinas como integración de tradiciones}

Bajo una perspectiva que reconoce la fragmentación de la ciencia, la idea de la integración del conocimiento científico no puede limitarse a modelar las relaciones mediadas por teorías. Una reformulación del problema de la integración incluye necesariamente una ampliación de la perspectiva desde la cual atacamos dicho problema. La reformulación requiere, en particular, que nos refiramos a cuestiones tales como las interacciones materiales (técnicas, artefactos, sustancias) y sociales que se establecen entre tipos de tradiciones y entre tradiciones individuales. Este tipo de interacciones ocurre no sólo entre diferentes disciplinas sino, fundamentalmente, en el proceso de formación y consolidación de una disciplina. Así pues, en el modelo de integración de la ciencia que aquí propongo se resuelve la aparente paradoja que Bechtel (1993) ha señalado: la integración del conocimiento

12 Algunas prácticas que permiten la construcción de objetos sin mediación teórica (en el sentido de un conjunto de enunciados o modelos a partir de los cuales se infieren explicaciones o predicciones) se desenvuelven, por ejemplo, en la construcción y evolución de técnicas experimentales. Si bien en ese proceso puede partirse de o desarrollarse un conjunto de aproximaciones o modelos del comportamiento de un grupo de fenómenos, no siempre estos modelos constituyen una teoría acerca de cómo y porqué debe funcionar un cierto artefacto tecnológico. Así, el desarrollo de la teoría sobre la cual se apoyaba la microscopía electrónica en las décadas de los años treinta a los cincuenta, no permitía inferir que fuera posible observar macromoléculas (como el DNA), algo que sin embargo ocurríb. Asimismo, ni esta teorla ni ninguna otra (de carácter bioquímico o químico) permiten explicar - aún hoy en día- las maneras en que diferentes compuestos y tinciones, bajo ciertas condiciones, interaccionan con las células y tejidos para proporcionar imágenes que son satisfactorias. Muchas de las sustancias químicas que se utilizan lo son, exclusivamente, debido a que se trataba de sustancias utilizadas ampliamente por la tradición de los microcopistas de luz (recuérdese que la microscopía de luz funciona bajo principios muy distintos a los de la microscopía electrónica). Ningún microscopista electrónico (o para el caso, ningún científico) podría dudar de que las técnicas de fijación, corte, tínción y exposición de especímenes biológicos utilizadas por los investigadores son parte del conocimiento incorporado en esa tradición. Véase Rasmussen (1993) para más detalles de este ejemplo y Ferguson (1993) para una perspectiva más general del pensamiento no verbal que es necesario en la construcción de tecnología. 
cientifico en disciplinas conduce a la desintegración de éste en disciplinas cada vez más especializadas. Pese a ello, en el centro de la imagen fragmentada de la ciencia y de la amenaza de la balcanización, el tema de la conformación de nuevas disciplinas científicas, así sea un proceso que en parte promueve la especialización, cobra importancia y se transforma en un reto en los estudios sobre la ciencia. Lenoir (1993) ha dicho al respecto que la "suave integración de los diferentes aspectos de la ciencia, que se daba como un hecho en los enfoques en que dominan las teorías, se convierte ella misma en objeto de investigación [...] Dentro del complejo de problemas generados por la disgregación de la ciencia, la disciplina emerge como un sitio crucial" (p. 71).

El reto de la fragmentación de la ciencia puede enfrentarse caracterizando las disciplinas como estructuras que, bajo condiciones históricas y sociales propicias, surgen y se estabilizan a través de la construcción de interacciones entre los diferentes tipos de tradiciones en que se organiza una comunidad. Ahora bien, en la idea de disciplinas científicas que me interesa defender hay dos aspectos fundamentales. Por un lado, las disciplinas deben verse como estructuras que resultan de y constituyen el medio ambiente en el cual tiene lugar la interacción de diferentes tipos de tradiciones científicas alrededor de un dominio compartido. ${ }^{13}$ Por otro, el conocimiento de una disciplina -cuya integración es un hecho histórico, y no lógico, alrededor de un dominio específico - proviene de la manera como se integran diferentes tradiciones científicas en un marco institucional. ${ }^{14}$

Ambos aspectos se encuentran, sin embargo, íntimamente relacionados; su distinción se hace exclusivamente con el fin de analizarlos.

En efecto, en una disciplina la reproducibilidad de las tradiciones requiere de recursos material-sociales, y las instituciones facilitan la disponibilidad de esos recursos. Ese marco institucional se construye bajo circunstancias históricas y sociales que no tienen porqué verse como si estuvieran disociadas de los procesos mismos de construcción de conocimiento, ya que la reproducibilidad de las tradiciones requiere tanto de la transmisión de

13 La cuestión de qué tipos de tradiciones interaccionan en una disciplina particular es algo que debe responderse empíricamente, como resultado de un análisis histórico. Hay disciplinas en que predominan tradiciones de tipo experimental como la biología celular (véase Bechtel, 1993), o disciplinas donde predominan tradiciones de tipo descriptivista como la taxonomía (véase Mayr, 1982) o disciplinas en donde proliferan una diversidad de tipos de tradiciones como la evolución molecular(véase Suárez, 1996).

14 Lenoir (1993) ha defendido una distinción paralela a ésta. Según él es necesario distinguir entre el "programa de investigación" de una disciplina (esto es, el conjunto de prácticas y objetos con los que se lleva a cabo la investigación en tomo a un conjunto de problemas), y el "programa disciplinario" como estrategia de conformación institucional de una disciplina (esto es, su lugar en la red de relaciones sociales). 
prácticas y habilidades mediante la educación, como de la sujeción de los miembros de esa tradición a procesos de revisión que operan bajo criterios de calidad específicos de la disciplina. Ambos procesos se dan en el marco de diferentes instituciones sociales (departamentos de investigación, sociedades, congresos, revistas reconocidas), muchas de las cuales son instituciones estrictamente disciplinarias. De hecho, entre los factores que contribuyen a la construcción de instituciones disciplinarias se encuentran las interacciones entre las tradiciones que la conforman y entre éstas y otras disciplinas. Veamos un ejemplo.

Como señalé antes, un caso que he estudiado en otro lugar y que ilustra lo anterior es el de la evolución molecular, una disciplina biológica que nace en los inicios de la década de los sesenta y se consolida en la siguiente década (véase Suárez, 1996). La evolución molecular es un caso interesante desde varios puntos de vista, uno de los cuales es el de la diversidad de prácticas y fines epistémicos que conviven en ella. Los evolucionistas moleculares se dedican, por mencionar sus actividades más comunes, a la elaboración de modelos teórico-matemáticos de genética de poblaciones, a la construcción de filogenias (clasificaciones) moleculares, al desarrollo de nuevas técnicas experimentales y a la estabilización de fenómenos en sus laboratorios. De hecho, el nombre mismo de la disciplina refleja la "reconciliación" de las dos grandes ramas que han caracterizado la biología desde sus orígenes: la biología evolutiva y la biología experimental (véase Mayr, 1982).

En los inicios de la década de los sesenta esta diversidad de fines epistémicos se expresaba en diferentes proyectos de investigación de un cierto número de científicos (lo que podríamos llamar tradiciones individuales), cuyo denominador común era el interés por aplicar o desarrollar técnicas moleculares en el estudio de la evolución biológica. En esos años era notoria, sobre todo, la autonomía relativa entre distintos tipos de esas tradiciones. Por ejemplo, los objetivos de varias tradiciones experimentales se articularon, a finales de la década de los sesenta, en la construcción del fenómeno del DNA satélite. ${ }^{15}$ En ese proceso de construcción de conocimiento experimental intervinieron los esfuerzos de al menos tres grupos de investigación (tradiciones individuales): el grupo que desarrolló las primeras técnicas de hibridación de ácidos nucleicos utilizadas en ese proyecto (Spiegelman y Hall, de la Universidad de Urbana), el grupo que caracterizó algunas de las variables importantes de esas técnicas (Marmur, Doty, Lane y Schilkdraut,

15 El DNA-satélite es una fracción del material genético de las células de organismos eucariontes (plantas, animales, hongos y protoctistas) que consiste en segmentos de tamaño mediano (unos 400 nucleótidos de longitud) que se encuentran repetidos millones de veces. Con respecto al significado de la expresión "construcción del DNA-satélite" véase Suárez (1996). 
de la Universidad de Harvard) y, por supuesto, el grupo que finalmente estabilizó el nuevo fenómeno (Britten, Waring y Kohn del Instituto Carnagie de Washington). Estos tres grupos sostenían estrechas relaciones entre sí. Intercambiaban no sólo materiales (sustancias químicas y biológicas), información y técnicas, sino también investigadores y estudiantes. Sin embargo, sus interacciones con tradiciones de otro tipo (teóricas o descriptivistas), en estos años, eran prácticamente nulas; sus intereses o fines epistémicos eran hasta entonces sumamente distintos. Relaciones similares ocurrieron a lo largo de la década de los sesenta entre diferentes tradiciones descriptivistas (ocupadas todas con el objetivo de construir filogenias con las nuevas técnicas moleculares) y teóricas (ocupadas en la elaboración de modelos adecuados a los nuevos datos de variabilidad genética descubiertos con las nuevas técnicas moleculares).

Sin embargo, hacia finales de los años sesenta las relaciones entre los grupos de investigación interesados en la aplicación de técnicas moleculares a la solución de problemas evolutivos ya involucraban intercambios de todo tipo, los cuales promovieron relaciones entre distintos tipos de tradiciones. Tales intercambios involucraban la transferencia de información, de materiales, de técnicas, de conceptos y de modelos teóricos. Esto no borró, sin embargo, la relativa autonomía entre los distintos tipos de prácticas que caracterizaban cada tipo de tradición. Como señalé antes, los tipos de prácticas constituyen capacidades cognitivas articuladas en torno a diferentes objetos o fines epistémicos; los métodos (que incluyen tipos de criterios y capacidades), así como los instrumentos desarrollados en torno a la elaboración de clasificaciones, por ejemplo, no se utilizan para alcanzar los mismos objetivos que los métodos e instrumentos de tradiciones experimentales, a pesar de que los nuevos descriptivistas utilicen, con frecuencia, los datos obtenidos con nuevas técnicas experimentales.

La construcción de diferentes tipos de objetos científicos (modelos matemáticos, clasificaciones, técnicas y fenómenos) constituye el proceso mediante el cual se construye el dominio de una disciplina, aspecto en el que abundaré en la siguiente sección. Lo que aquí quiero señalar es que la construcción de un dominio propio de la evolución molecular requirió no sólo de un intenso intercambio conceptual y material entre tradiciones, sino de un intenso programa de organización social, que fue la base sobre la cual se conformaron algunas de las instituciones características de esa disciplina. Me refiero, por ejemplo, a los debates que de manera coordinada unieron a los nuevos evolucionistas moleculares frente a los evolucionistas tradicionales, ${ }^{16}$ así como a la creación de estructuras sociales que promueven

16 Dos de esos debates fueron el de la superioridad de los datos moleculares sobre los datos paleoantropológicos en la determinación del tiempo de divergencia del hombre y el chimpancé 
y evalúan la reproducción de las prácticas desarrolladas por una comunidad en torno a un grupo de objetos. La creación de departamentos de enseñanza e investigación en las universidades o la publicación de revistas especializadas con un consejo editorial prestigioso (en este caso, integrado por los fundadores de la disciplina y algunos científicos miembros de disciplinas establecidas) ejemplifican este punto. ${ }^{17}$ Tales estructuras sociales promueven la integración de tradiciones, y por tanto de conocimiento, en el sentido amplio al que me he referido; esto es, facilitan los intercambios no exclusivamente teóricos entre los miembros de una disciplina, al tiempo que regulan y evalúan las interacciones - y la posible integración - entre distintas disciplinas. Me interesa ahora, sin embargo, centrarme en un aspecto de particular relevancia filosófica para la reformulación del problema de la integración de la ciencia: el concepto de dominio de una disciplina.

\section{La consolidación de una disciplina. El concepto de dominio}

Como señalé al referirme al trabajo de Darden y Maull (1977), el concepto de dominio de Shapere (1984), que originalmente se refería al dominio de teorías, puede generalizarse para referirse al dominio de una disciplina. Según Shapere, el cuerpo de información que constituye el dominio es un conjunto de elementos (items) que se toman como hechos en ese campo, pero que no se restringen a las meras observaciones. Lo que en una etapa del conocimiento era una "teoria" que resolvía un problema, en otra se convierte en parte del dominio que es investigado y al cual se busca explicar. "Lo que era una 'teoría' se convierte en un hecho, pero un 'hecho' respecto al cual hay un problema", dice Shapere (op. cit., p. 282). ${ }^{18}$ Por otra parte, en la caracterización de Shapere los elementos de un dominio

y el de los neutralistas (moleculares) contra los seleccionistas (predominantemente biólogos de organismos, véase Suárez, 1996).

17 Por ejemplo, la primera página del primer número del Journal of Molecular Evolution, publicada en 1971, expresaba los intereses de los miembros de la nueva disciplina y en ese sentido marcaba los límites del dominio de lo que entonces ya se llamaba evolución molecular: "This journal publishes articles in the following research fields: (1) Biogenetic evolution (prebiotic molecules and their interaction). (2) Evolution of informational macromolecules (primary through quaternary structure). (3) Evolution of genetic control mechanisms. (4) Evolution of enzyme systems and their products. (5) Evolution of macromolecular systems (chromosomes, mitochondria, membranes, etc.). (6) Evolutionary aspects of molecular population genetics."

18 En 1991, Darden define también asf el dominio: "The domain is a set of items, which, on the one hand, may be data produced by observations or experiments. On the other hand, domain items may be general claims that have previously been labeled 'empirical laws' or 'explanatory theories'; they become part of the domain when they are judged to be well confirmed and are themselves in need of explanation" (Darden, 1991, p. 17). 
no se encuentran aislados unos de otros sino relacionados en un cuerpo de información respecto al cual hay un problema; frecuentemente ese problema consiste, precisamente, en describir y explicar las relaciones entre los elementos (items) del dominio. La investigación, pues, se refiere a los elementos en relación y no a "hechos" aislados.

Ambos aspectos de la caracterización de Shapere son relevantes para hablar del dominio de una disciplina. Por un lado, los elementos y problemas por explicar en una disciplina no se restringen a meras observaciones; más aún, es claro que la idea de que en la ciencia existen diferentes tipos de tradiciones supone una visión de la construcción del conocimiento que no se limita a la oposición entre "teorías" y "observaciones", por lo que resulta natural reconocer que en el dominio de una disciplina se mezclen objetos de tipos tan diversos como modelos, regularidades, técnicas y fenómenos. Por otro lado, el énfasis de Shapere en que los elementos de un dominio se encuentran en relación, proporciona una explicación de la cohesión epistémica que caracteriza la integración de tradiciones en una disciplina.

Sin embargo, a diferencia de Shapere y de otros autores que han utilizado el concepto de dominio (como Darden y Maull, 1977 y Bechtel, 1993), quisiera hacer énfasis en que al referirme al dominio de una disciplina me refiero al resultado (en un momento dado) de un proceso histórico de construcción de conocimiento. El dominio, en mi modelo, es parte importante de una explicación diacrónica de cómo ocurre la integración de conocimiento a través de la formación de disciplinas y de cómo se conforma el marco institucional que comparte un grupo de tradiciones. Ambos aspectos, como vimos en la sección anterior, se encuentran estrechamente relacionados.

Así pues, el dominio de una disciplina no es un conjunto fijo y común de elementos respecto a los cuales exista un grupo de problemas que compartan diferentes tradiciones. En primer lugar, porque las diferentes tradiciones que se integran en una disciplina conservan un dominio relativamente propio; solamente algunos de los elementos del dominio son comunes a varias de las tradiciones que integran esa disciplina; una cierta fragmentación de la ciencia se conserva aún en el seno de una disciplina, como lo ilustra hoy en día la organización de la investigación en la evolución molecular. ${ }^{19} \mathrm{En}$

19 Me refiero al hecho de que, a pesar de que exista un dominio compartido por varios tipos de tradiciones, los tipos de problemas que surgen en los diferentes tipos de tradiciones requieren diferentes tipos de prácticas. Así, en el laboratorio del doctor Francisco Ayala (de la Universidad de California, Irvine, UCI) una parte importante de las actividades, sean experimentales o conceptuales, se centran en la construcción de modelos matemáticos que describen la dinámica de la variabilidad genética (un problema típico de tradiciones teóricas). Mientras que en el laboratorio del doctor Walter Fitch (también de la UCI) las actividades (también experimentales o conceptuales) se centran en la construcción de clasificaciones naturales (un problema típico de las tradiciones descriptivistas). Ambos son miembros destacados de 
segundo lugar, la construcción de conocimiento significa que continuamente se incorporan nuevos elementos, mientras que otros dejan de ser considerados relevantes. Todo lo cual genera nuevas condiciones para la construcción de otros elementos y transforma las relaciones entre ellos (por ejemplo, su importancia relativa). Es en este sentido que una reconstrucción racional de la estructura del dominio de la disciplina no parece posible ni relevante; para cada tipo de tradición, en un contexto dado, existe una determinada relación de los elementos del dominio que es problemática y que no necesariamente comparte con otros tipos de tradición o en otro contexto o momento histórico-social.

Ahora bien, pese a ese carácter siempre fluido y cambiante del dominio, es posible realizar una caracterización básica del proceso mediante el cual un dominio se construye y, con ello, permite la cohesión epistémica de un grupo de tradiciones. Esto se ilustra en el caso de la evolución molecular(Suárez, 1996), en el que he localizado tres etapas básicas del proceso de construcción del dominio que parecen poder extrapolarse a otros casos de la historia de la ciencia. Estas tres etapas son:

a) la apertura de un nivel de organización a la investigación, gracias a la utilización de nuevas técnicas experimentales (en este caso, la apertura, en la primera mitad de la década de los sesenta, del nivel de organización molecular -esto es, las llamadas moléculas informacionales, ácidos nucleicos y proteínas- a la investigación evolutiva gracias al desarrollo de la biología molecular y la bioquímica),

b) el establecimiento de nuevos elementos en un dominio propio (por ejemplo, el desarrollo de los conceptos de reloj molecular y de restricciones funcionales en 1963-1965, y la determinación de la elevada variabilidad genética de las poblaciones naturales en 1966) y

c) la formulación de problemas relativos a las relaciones entre los elementos del nuevo dominio (por ejemplo, en 1968-1969 la publicación de la teoría teutral, que relacionaba los elementos de la segunda etapa en una nueva explicación de la evolución en el nivel molecular, o la idea (a partir de 1969) de que las moléculas informacionales y el reloj molecular permiten la construcción de mejores filogenias.

Las etapas anteriores corresponden a la profundización de las interacciones entre distintos tipos de tradiciones en la conformación de la evolución molecular. En la primera etapa cada tipo de tradición tiende a enfocarse a resolver problemas para los cuales no es necesaria, en buena medida, 
la cooperación con otros tipos de tradiciones, aunque los intercambios con tradiciones de su mismo tipo es frecuente. El incremento en los intercambios y en la utilización de nuevas técnicas para resolver problemas tradicionales o novedosos generalmente es el contexto en el cual se estabilizan nuevos elementos del dominio en formación, lo que constituye la segunda etapa en la construcción de las interacciones entre tradiciones. Por último, las diferentes maneras en que se relacionan esos elementos generan el tipo de problemas que marcan la tercera etapa de construcción del dominio de una disciplina; generalmente, esos problemas requieren de las prácticas y el enfoque coordinado pero distinto de diferentes tipos de tradiciones.

La autonomía relativa que existe entre diferentes tipos de tradiciones cientificas es más visible antes de que éstas se integren alrededor de un dominio. Una vez integradas alrededor de un grupo de elementos, las tradiciones tienden a cooperar en la solución de los diferentes problemas que atañen a las relaciones entre los elementos del dominio de la disciplina. Sin embargo, las tradiciones subsisten en esta etapa en la medida en que siguen existiendo diferentes tipos de problemas y preguntas que requieren diversos tipos de prácticas y habilidades para su solución. Por ejemplo, el reloj molecular es un elemento del dominio de las tradiciones teóricas en la medida en que la teoría neutral puede o intenta explicar su existencia y, por tanto constituye una evidencia en contra de la acción de la selección natural en el nivel molecular; pero ese mismo concepto de reloj molecular constituye una regularidad útil en las tradiciones descriptivistas, en donde permite calcular tiempos de divergencia entre especies en la elaboración de filogenias. En efecto, las relaciones entre los elementos del dominio generan diferentes tipos de problemas en las diferentes tradiciones. Este es el núcleo de mi propuesta de reformulación del problema de la integración de la ciencia.

Se trata de un modelo en el que, a la par que se reconoce la naturaleza fragmentada de la ciencia, se propone que las interacciones que de facto generan la integración son de tipo material, social y cognitivo (pero no exclusivamente teórico). Tales interacciones tampoco se reducen a lo "meramente social"; sus connotaciones epistémicas se expresan en el concepto de dominio y en la cohesión cognitiva que éste genera al centrar un grupo de tradiciones alrededor de un conjunto compartido de elementos. La integración de la ciencia no se reduce a la integración de sus resultados (sean teorías más o menos universales o modelos locales). La integración es un asunto que atañe a los objetos mismos de la investigación. Pese a la fragmentación de los objetivos y de las habilidades que constituyen el conocimiento de la ciencia, en circunstancias favorables como las que prevalecen en una disciplina consolidada esa diversidad se centra alrededor de un dominio compartido. Ésa constituye la respuesta a la pregunta de 
en qué sentido, bajo la imagen fragmentada de la ciencia, puede decirse que el conocimiento científico constituye un cuerpo unificado y racional de creencias.

\section{Conclusiones}

Las disciplinas científicas constituyen estructuras tanto sociales como cognitivas que agrupan una comunidad en torno de un grupo relativamente estable pero cambiante de problemas. Los elementos (fenómenos, regularidades, modelos, etc.) que forman parte de su dominio, así como sus instituciones sociales son barreras o fronteras que las separan de otras estructuras similares. Bechtel (1993) ha señalado, me parece que correctamente, el carácter paradójico de la conformación de disciplinas. El origen de una disciplina es, por un lado, el resultado de la integración de diferentes tipos de prácticas (tradiciones), pero por otro lado es también el resultado de la especialización o desintegración de la ciencia en disciplinas con dominios restringidos.

La proliferación de disciplinas, sin embargo, no necesariamente opera en sentido contrario a la integración de la ciencia. El modelo de integración del conocimiento que he presentado, a diferencia de los modelos de unificación teórica de la ciencia, se refiere a la articulación local de prácticas y objetos provenientes de diferentes tradiciones, cuyo objeto consiste en abordar problemas complejos. La integración de tipos de prácticas en una disciplina es, más que un ideal, un recurso fructifero de la ciencia para la solución de problemas y no conduce ni pretende llevar a la creación de una sola disciplina o de una sola teoría universal. Tal integración, pues, no puede verse como de tipo "meramente social" o "cultural". Es en ese sentido que he introducido el concepto de dominio de una disciplina, el cual constituye el resultado de la cohesión cognitiva de un grupo de tradiciones en una disciplina. Las connotaciones epistémicas de este concepto son, sin embargo, más amplias y se elaborarán en un trabajo posterior. 


\section{BIBLIOGRAFIA}

Bechtel, W., 1993, "Integrating Sciences by Creating New Disciplines: The Case of Cell Biology", Biol. and Phil, vol. 8, no. 3, pp. 277-299

Bijker, W.E., T.P. Hugues y T.J. Pinch (eds.), 1987, The social construction of Technological Systems. New Directions in the Sociology and History of Technology, MГT Press, Cambridge, Mass.

Burian, R.M., 1993, "Unification and Coherence as Methodological Objectives in the Biological Sciences", Biol. and Phil, vol. 8, no. 3, pp. 301-318.

- 1993a, "Technique, Task Definition and the Transition from Genetics to Molecular Genetics; Aspects on the Work on Protein Synthesis in the Laboratories of J. Monod and P. Zamecnik", J. Hist. of Biol., vol. 26, no. 3, 387-407.

Collins, H., 1988, Changing Order: Replication and Induction in Scientific Practice, Sage, Londres-Los Ángeles.

Darden, L., 1991, Theory Change in Science, Oxford University Press, Nueva YorkOxford.

Darden, L. y N. Maull, 1977, "Interfield Theories", Phil. of Science, no 43, pp. 121164.

Desmond, A., 1989, The Politics of Evolution, The University of Chicago Press, Chicago Illinois.

Dupré, J., 1993, The Disorder of Things, Harvard University Press, Cambridge, Mass. Fergusosn, E.S., 1993, Engineering and the Mind's Eye, MIT Press, Cambridge, Mass. Galison, P., 1987, How Experiments End, The University of Chicago Press, Chicago. Gaudillière, J., 1993, "Molecular Biology in the French Tradition? Redefining Local Traditions and Disciplinary Patterns", Journal of the History of Biology, vol. 26, no. 3, pp. $473-498$.

Gooding, D., T. Pinch y S. Schaffer (eds.), 1989, The Uses of Experiment. Studies in the Natural Sciences, Cambridge University Press, Cambridge.

Hacking, I., 1983, Representing and Intervening, Cambridge University Press, Cambridge, Mass.

_ 1992 , “'Style' for Historians and Philosophers”. Stud. Hist. Phil. Sci., vol. 23, no. 1, pp. 1-20.

Hull, D.L., 1972, "Reduction in Genetics: Biology or Philosophy?", Philosophy of Science, no. 39, pp. 491-498.

__, 1974, Philosophy of Biological Science, Prentice Hall, Englewood-Cliffs.

Kitcher, P., 1984, "1953 and all That: A Tale of Two Sciences". Philosophical Review, no. 93, pp. 355-373.

Latour, B., 1987, Science in Action, Harvard University Press, Cambridge, Mass.

Latour, B. y S. Woolgar, 1979, Laboratory Life, Princeton University Press, Princeton, Nueva Jersey.

Lenoir, T., 1993, "The Discipline of Nature and the Nature of Disciplines", en MesserDavidow, E., D.R. Shumway y Silvan, D.J. (eds.), Knowledges. Historical and Critical Studies in Disciplinarity, University Press of Virginia, Londres y Charlotte Ville, pp. 70-102. 
Martínez, S.F, 1993a, "Método, evolución y progreso en la ciencia (primera parte)", Crítica, vol. 25, no. 73, pp. 37-69.

—, 1993b, "Método, evolución y progreso en la ciencia" (segunda parte)", Crítica, vol. 25, no. 74, pp. 3-21.

— 1995 , "La autonomía de las tradiciones experimentales como problema epistemológico", Crítica, vol. 27, no. 80, pp. 3-48.

Mayr, E., 1982, The Growth of Biological Thought, The Belknap Press, Harvard University Press.

Nagel, E., 1961, The Structure of Science, Harcourt, Nueva York.

Pickering, A., 1989, "Living in the Material World", en Gooding et al., 1989, op cit., pp. 275-298.

Pickering, A. (ed.), 1992, Science as Practice and Culture, The University of Chicago Press, Chicago.

Rasmussen, N., 1993, "Facts, Artefacts and Nesossomes: Practicing Epistemology with the Electron Microscope", Stud. Hist. Phil. Sci., vol. 24, no. 2, pp. 227-265.

Rosenberg, R., 1994, Instrumental Biology and the Disunity of Science, The University of Chicago Press, Chicago.

Ruse, M., 1973, The Philosophy of Biology, Hutchison University Library, Londres.

Sarkar, S., 1992, "Models of Reduction and Categories of Reductionism", Synthese, no. 91, 1992, pp. 167-194.

Schaffner, K.F., 1967, "Approaches to Reduction", Philosophy of Science, no. 34, pp. 137-147.

-, 1993, "Theory Structure, Reduction and Disciplinary Integration in Biology", Biol. and Phil., vol. 8, no. 3, pp. 319-347.

Shapere, D., 1974 [1984], "Scientific Theories and Their Domains", en Shapere, D. 1984, Reason and the Search for Knowledge, Reidel, Dordrecht.

Shapin, S. y S. Schaffer, 1985, Leviathan and the Air Pump, Princeton University Press, Princeton.

Suárez, E., 1996, "El origen de disciplinas como integración de tradiciones: el caso de la evolución molecular", tesis de doctorado, Facultad de Ciencias, UNAM, México.

Vicedo, M., 1995, "Scientific Styles: Toward some common Ground in the History Philosophy, and Sociology of Science" en Perspectives on Science, vol. 3, no. 2, pp. 231-254.

Wimsatt, W., 1976a (1984), "Reductive Explanation: A Functional Account", en R.S. Cohen et al. (eds.), PSA, 1974, pp. 671-710. Publicado también en Sober, E. (ed.), 1984, Conceptual Išsues in Evolutionary Biology, MIT Press, Cambridge, pp. 477-507

- 1976b, "Reductionism, Levels of Organizations and the Mind Body Problem", en G. Globus, 1. Sabodnik y G. Maxwell (eds.), Conciousness and the Brain, Plenum, Nueva York, pp. 199-267. Waters, K., 1990, "Why the Antireductionist Consensus Wont Survive the Case of Classical Mendelian Genetics", PSA, 1990, vol. l, pp. 125-139. 